\title{
Precipitation in an Irradiated 625 Plus Alloy
}

\author{
Li-Jen $\mathrm{Yu}^{1}$ and Emmanuelle Marquis ${ }^{1}$
}

1. Department of Materials Science and Engineering, University of Michigan, Ann Arbor, MI

Alloy 625 plus is a highly-corrosion-resistant, nickel-based alloy that can be age-hardened to high strength levels without warm or cold working [1]. The existing nickel-based alloy used for nuclear applications such as Alloy 625 has excellent corrosion resistance. However, Alloy 625 needs to be cold worked in order to obtain high strength level, which is very difficult for large sized workpieces. On the other hand, Alloy 718 can be age-hardened but has insufficient corrosion resistance in severer environment. Thus, the development of Alloy 625 plus is to combine the advantage of corrosion resistance (more corrosion resistant than Alloy 718) and high strength of Alloy 625 with an agehardening capability similar to that of Alloy 718 [2]. With these superior properties, Alloy 625 plus would be considered as a candidate for nuclear applications. However, the microstructural changes of Alloy 625 plus under irradiation have not been revealed. To fill this gap, the precipitation behavior of Alloy 625 plus was investigated using transmission electron microscopy (TEM) and atom probe tomography (APT).

Alloy 625 plus was solution treated at $1100{ }^{\circ} \mathrm{C}$ for 1 hour, followed by water quenching. Samples were electropolished and then proton- or ion- irradiated at the Michigan Ion Beam Laboratory for a range of doses, temperatures, and dose rates. TEM and APT specimens were prepared using an FEI Nova 200 Nanolab focus ion beam (FIB). APT specimens were fabricated by utilizing concentric annular milling with a series of decreasing ion currents at $30 \mathrm{kV}$ applied voltage and a $5 \mathrm{kV}$ final milling step. The depth from sample surface was carefully controlled to be in the region of uniform irradiation damage level. Data was collected using a Cameca LEAP 4000X HR operated in laser mode using a $50 \mathrm{pJ}$ pulse energy, $200 \mathrm{kHz}$ pulse rate, and a target detection rate of $0.5 \%$ at $50 \mathrm{~K}$. Reconstructions were performed using the IVAS 3.6.12 software. TEM observations were performed on a JEOL 3011 high resolution electron microscope.

Solute clustering is evident after proton irradiation to $1.5 \mathrm{dpa}$ at $300{ }^{\circ} \mathrm{C}$. As illustrated in Figure 1 (a), the normalized difference between the experimental and random $10^{\text {th }}$ nearest neighbor distributions for Fe, $\mathrm{Nb}$, Ti and $\mathrm{Al}$ shows clear clustering. While these clustering tendencies are relatively faint, they are nonetheless significant when compared with the solution treated condition.

Minor or impurity elements such as $\mathrm{Si}, \mathrm{B}$ and $\mathrm{P}$ were also found co aggregated, as shown in figure 1 (b). Additionally, small regions exhibiting local ordering of $\mathrm{Ni}$ and $\mathrm{Cr}$ are observed (Figure 2). We will discuss the evolution of these microstructural features with dose and the differences between ion and proton irradiation behavior.

\section{References:}

[1] Frank, R.B. in "Custom Age 625 Plus Alloy - A Higher Strength Alternative to Alloy 625," Superalloys 718, 625, and Various Derivatives, ed. Loria, E.A., (TMS Publication, Warrendale, PA, 1991) p. 879. 
[2] Schmidt, N.B., DeBold, T.A. and Frank, R.B. Journal of Materials Engineering and Performance 1 (1992), p. 483.

[3] The authors acknowledge funding from the Department of Energy - Nuclear Energy University Program grant DE-NE0008423 and technical support from the Michigan Ion Beam Laboratory and the Michigan Center for Materials Characterization.
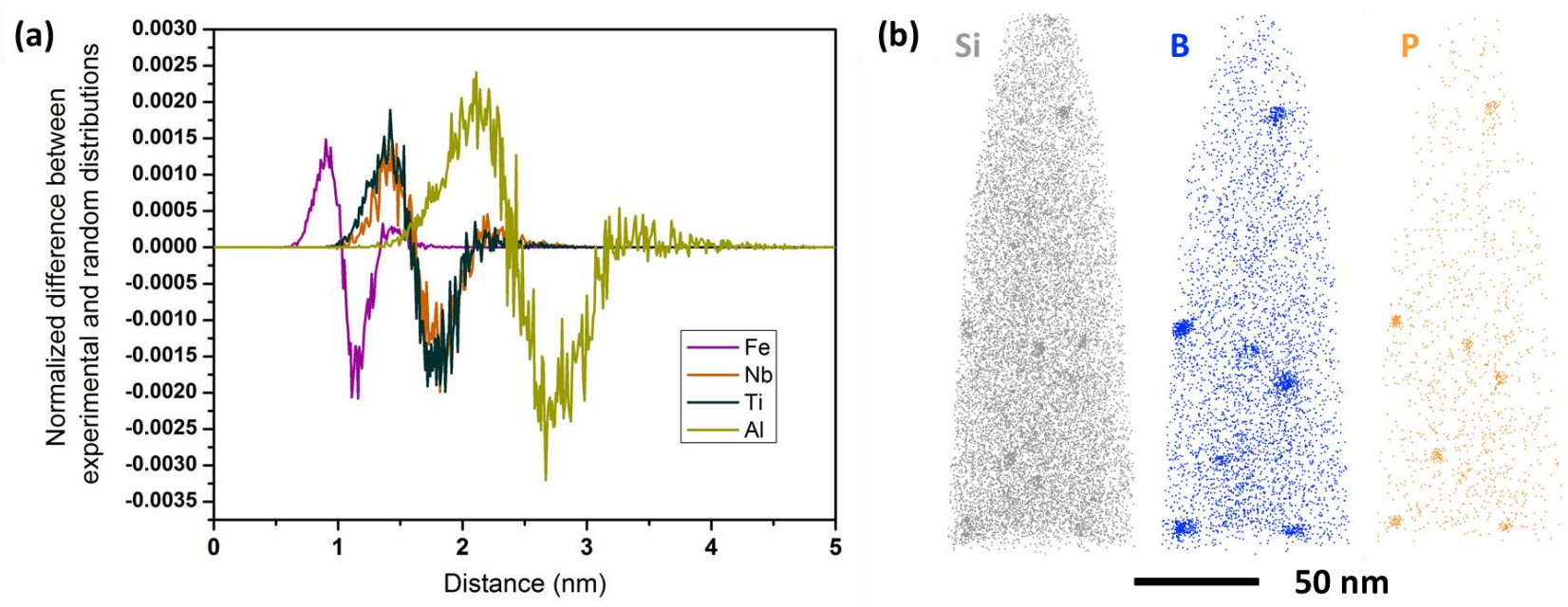

Figure 1. (a) Normalized difference between experimental and random $10^{\text {th }}$ nearest neighbor distributions of selected elements showing clustering after $2 \mathrm{MeV}$ proton irradiation to $1.5 \mathrm{dpa}$ at $300{ }^{\circ} \mathrm{C}$. (b) Atom maps showing the clustering of minor elements Si, B and P after irradiation.
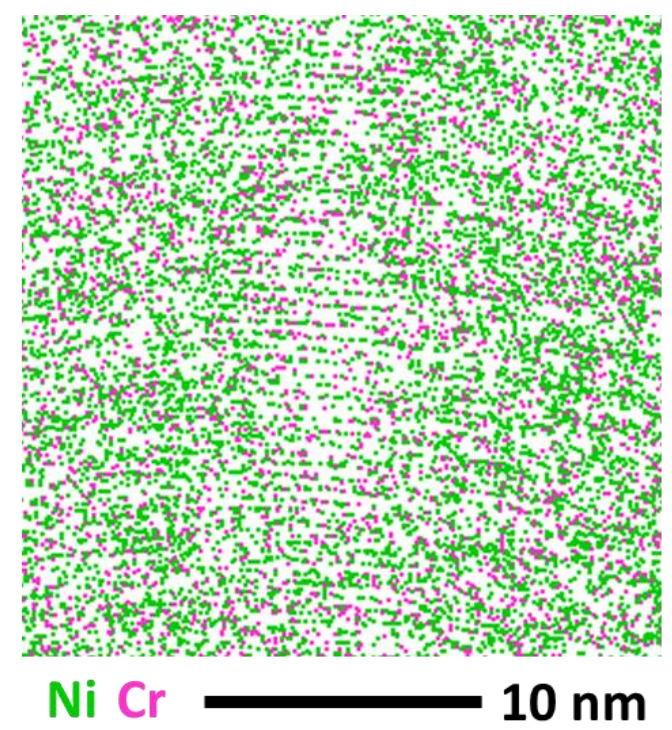

Figure 2. $2 \mathrm{~nm}$ thick slice atom maps showing local ordering after proton irradiation. 